\title{
Negative valuation of undesirable outputs in evaluating the efficiency of production systems
}

\author{
Chiang $\mathrm{Kao}^{1, *}$ \\ ${ }^{1}$ Department of Industrial and Information Management, National Cheng Kung University, Tainan, Taiwan, Republic of China
}

\begin{abstract}
This paper extends a data envelopment analysis (DEA) model that considers only desirable outputs in measuring efficiencies to incorporate undesirable outputs by requiring the associated multipliers to be negative. It is shown that the proposed model is the ratio-measure counterpart of the difference-measure model existed in the literature. It has the technology of variable returns to scale, and the modification made to it means that it also has the property of null-jointness, satisfies the axiom of weak disposability, and has positive shadow prices, considered as pitfalls in modeling undesirable outputs. In addition to the economic interpretation, the ratio-measure of efficiency of the proposed model better shows the relative performance of a unit than the difference-measure does, and its simpler form also provides a clearer geometric interpretation of efficiency measurement with undesirable outputs.
\end{abstract}

\section{Introduction}

An output is considered as undesirable in the production process if more of it being produced from the same amount of input is not wanted. Typical examples of undesirable outputs are waste water and pollutants generated in producing the main, desirable products. As environmental issues are becoming greater concern, the number of studies on undesirable outputs has grown. How to appropriately measure the efficiency of a production unit that produces both desirable and undesirable outputs using the data envelopment analysis (DEA) technique has also attracted numerous studies. Leleu [1] reviewed many articles on the modeling of undesirable outputs, highlighted some problems that can arise, and proposed a model that avoids these. This model also provides an economic interpretation of the production process.

In this note a DEA model extended from the BCC model of Banker et al. [2] that incorporates undesirable outputs by assigning negative values to the associated multipliers is proposed. This model is shown to be a ratio-measure counterpart of the difference-measure model of Leleu [1], maintaining the strengths of this while in a simpler form. Moreover, the ratio-measure of efficiency used in this work, in the range of zero and one, provides a better idea of the relative performance of a production unit than the difference-measure, in the range of zero and an unknown upper bound, does.

In the next section we will introduce the extended BCC model, in ratio form, and discuss its relationship with Leleu's model. Then, in Section 3, the properties of this model will be explored from the envelopment form. Finally, some conclusions will be drawn in Section 4 from the discussion of this model.

\section{Ratio model}

Banker et al. [2] proposed a DEA model, usually referred to as the BCC model, to measure the relative efficiency of a set of $n$ production units that apply multiple inputs $X_{i j}, i=1, \ldots, m$, to produce multiple outputs $Y_{r j}, r=1, \ldots, m$, under the technology of variable returns to scale. All the outputs are assumed to be desirable. The output model, in ratio form, is as follows:

$$
\begin{aligned}
\frac{1}{E_{0}}=\min . & \frac{\sum_{i=1}^{m} v_{i} X_{i 0}+v_{0}}{\sum_{r=1}^{s} u_{r} Y_{r 0}} \\
\text { s.t. } & \frac{\sum_{i=1}^{m} v_{i} X_{i j}+v_{0}}{\sum_{r=1}^{s} u_{r} Y_{r j}} \geq 1, j=1, \ldots, n \\
& u_{r}, v_{i} \geq 0, r=1, \ldots, s, i=1, \ldots, m \\
& v_{0} \text { unrestricted in sign }
\end{aligned}
$$

where the multipliers $u_{r}$ and $v_{i}$ can be considered as the values of the corresponding outputs and inputs, respectively, as perceived by the decision maker, and $E_{0}$ is the efficiency of the unit being evaluated.

Suppose this model is extended to include undesirable outputs $B_{k j}, k=1, \ldots, t$. Since these outputs are undesirable, the values perceived by the decision maker must be negative. We use $-w_{k}$, where $w_{k} \geq 0$, to represent the multiplier of the undesirable output $B_{k}$. The extended BCC model, with undesirable outputs, can thus be formulated as follows:

$$
\frac{1}{E_{0}}=\min . \frac{\sum_{i=1}^{m} v_{i} X_{i 0}+v_{0}}{\sum_{r=1}^{s} u_{r} Y_{r 0}+\sum_{k=1}^{t}\left(-w_{k}\right) B_{k 0}}
$$

\footnotetext{
*Corresponding author: ckao@mail.ncku.edu.tw
} 


$$
\begin{gathered}
\text { s.t. } \frac{\sum_{i=1}^{m} v_{i} X_{i j}+v_{0}}{\sum_{r=1}^{s} u_{r} Y_{r j}+\sum_{k=1}^{t}\left(-w_{k}\right) B_{k j}} \geq 1, j=1, \ldots, n \\
u_{r}, v_{i}, w_{k} \geq 0, r=1, \ldots, s, i=1, \ldots, m, k=1, \ldots, t
\end{gathered}
$$

$v_{0}$ unrestricted in sign

This model obviously has multiple solutions, and if $(\boldsymbol{u}, \boldsymbol{v}, \boldsymbol{w})$ is a solution, then so is $(c \boldsymbol{u}, c \boldsymbol{v}, c \boldsymbol{w})$ for $c>0$. Due to the nature of multiple solutions, it is conventional to set the denominator of the objective function to one as a normalization constraint, and to minimize the numerator alone. This is equivalent to applying the variable substitution technique of Charnes and Cooper [3] to transform a fractional program into a linear one. The resulting linear program, usually called the multiplier form, is:

$$
\begin{aligned}
1 / E_{0}=\min . & \sum_{i=1}^{m} v_{i} X_{i 0}+v_{0} \\
\text { s.t. } \quad & {\left[\sum_{r=1}^{s} u_{r} Y_{r j}+\sum_{k=1}^{t}\left(-w_{k}\right) B_{k j}\right]-\left(\sum_{i=1}^{m} v_{i} X_{i j}+v_{0}\right) \leq 0, } \\
j=1, \ldots, n & \\
& \sum_{r=1}^{s} u_{r} Y_{r 0}+\sum_{k=1}^{t}\left(-w_{k}\right) B_{k 0}=1 \\
& u_{r}, v_{i}, w_{k} \geq 0, r=1, \ldots, s, i=1, \ldots, m \\
\quad & \quad k=1, \ldots, t \\
& v_{0} \text { unrestricted in sign }
\end{aligned}
$$

At optimality, [ $\left.\sum_{r=1}^{s} u_{r} Y_{r 0}+\sum_{k=1}^{t}\left(-w_{k}\right) B_{k 0}\right]-\left(\sum_{i=1}^{m} v_{i} X_{i 0}+\right.$ $\left.v_{0}\right)=0$ is the production frontier corresponding to the unit being evaluated, provided the solution is unique. The terms in square brackets in constraint set (3.1) are the observed aggregate output, and the terms in parentheses are the aggregate output expected to be produced from the inputs of this unit, and their ratio is the output efficiency of this unit shown in the ratio model (2). Constraint set (3.1) states that when the multipliers selected by this unit to calculate its efficiency are applied to calculate the efficiency of any unit, they must be less than or equal to one to ensure that all units lie below the frontier of $\left[\sum_{r=1}^{s} u_{r} Y_{r 0}+\sum_{k=1}^{t}\left(-w_{k}\right) B_{k 0}\right]-\left(\sum_{i=1}^{m} v_{i} X_{i 0}+v_{0}\right)$ $=0$.

Following the notation of the extended BCC model, the model proposed by Leleu [1], in what he called dual form, can be formulated as:

$$
\min . \varphi
$$

$$
\begin{array}{ll}
\text { s.t. } & \left(\sum_{r=1}^{s} u_{r} Y_{r j}-\sum_{k=1}^{t} w_{k} B_{k j}-\sum_{i=1}^{m} v_{i} X_{i j}\right)-\left(\sum_{r=1}^{s} u_{r} Y_{r 0}\right. \\
& \left.-\sum_{k=1}^{t} w_{k} B_{k 0}-\sum_{i=1}^{m} v_{i} X_{i 0}\right) \leq \varphi, j=1, \ldots, n \\
& \sum_{r=1}^{s} u_{r} g_{r}^{Y}+\sum_{k=1}^{t} w_{k} g_{k}^{B}=1 \\
& \sum_{r=1}^{s} u_{r} Y_{r 0}-\sum_{k=1}^{t} w_{k} B_{k 0}+\varphi \geq 0
\end{array}
$$

$$
u_{r}, v_{i}, w_{k} \geq 0, r=1, \ldots, s, i=1, \ldots, m, k=1, \ldots, t
$$

where $\boldsymbol{g}=\left(\boldsymbol{g}^{Y}, \boldsymbol{g}^{B}\right)$ is a direction vector and $v_{i}, u_{r}, w_{k}$ are the shadow prices of the $i$ th input, $r$ th desirable output and $k$ th undesirable output, respectively. Leleu [1] provided an economic interpretation of this model. Constraint set (4.1) states that the difference between the profit of any Unit $j$ and the unit being evaluated, which is the profit inefficiency, must be less than or equal to $\varphi$, and the objective function (4.0) is to find the minimum profit inefficiency $\varphi$ of the focal unit. Constraint (4.2) is the conventional normalization constraint used to solve the problem of homogeneity of degree one of costs, revenues, and profits in dual prices. Constraint (4.3) requires the efficient revenue from the desirable and undesirable outputs to be positive. Finally, constraint set (4.4) restricts all the shadow prices to be nonnegative.

Denote $\varphi=\left(\sum_{i=1}^{m} v_{i} X_{i 0}+v_{0}\right)-\left[\sum_{r=1}^{s} u_{r} Y_{r 0}+\right.$ $\left.\sum_{k=1}^{t}\left(-w_{k}\right) B_{k 0}\right]$ as the difference between the expected aggregate output and the observed aggregate output, which is the output inefficiency in a difference form, and is the objective function of Leleu's model (4.0). This is in contrast to the ratio form of $1 / E_{0}=\left(\sum_{i=1}^{m} v_{i} X_{i 0}+v_{0}\right) /$ $\left[\sum_{r=1}^{s} u_{r} Y_{r 0}+\sum_{k=1}^{t}\left(-w_{k}\right) B_{k 0}\right]$ of the extended BCC model. If $\varphi=\left(\sum_{i=1}^{m} v_{i} X_{i 0}+v_{0}\right)-\left[\sum_{r=1}^{s} u_{r} Y_{r 0}+\sum_{k=1}^{t}\left(-w_{k}\right) B_{k 0}\right]$ is substituted into constraints (4.1) and (4.3), then $\left[\sum_{r=1}^{s} u_{r} Y_{r j}+\sum_{k=1}^{t}\left(-w_{k}\right) B_{k j}\right]-\left(\sum_{i=1}^{m} v_{i} X_{i j}+v_{0}\right) \leq 0$ and $\sum_{i=1}^{m} v_{i} X_{i 0}+v_{0} \geq 0$ are obtained, respectively. The former is constraint (3.1) of the extended BCC model. For the latter, since we have $\left(\sum_{i=1}^{m} v_{i} X_{i 0}+v_{0}\right) \geq$ $\left[\sum_{r=1}^{s} u_{r} Y_{r 0}+\sum_{k=1}^{t}\left(-w_{k}\right) B_{k 0}\right]=1$ in the extended BCC model, the condition of $\sum_{i=1}^{m} v_{i} X_{i 0}+v_{0} \geq 0$ is obvious. When the direction vector of $\boldsymbol{g}=\left(\boldsymbol{g}^{Y}, \boldsymbol{g}^{B}\right)$ is replaced with $\boldsymbol{g}=\left(\boldsymbol{Y}_{0},-\boldsymbol{B}_{0}\right)$, constraint (4.2) becomes $\sum_{r=1}^{s} u_{r} Y_{r 0}+$ $\sum_{k=1}^{t} w_{k}\left(-B_{k 0}\right)=1$, which is the same as constraint (3.2). Finally, constraint set (4.4) is the same as constraint set (3.3), and constraint (3.4) is specific to the extended BCC model, which has no effect on Leleu's model (4). In sum, the extended BCC model is equivalent to Leleu's model, except the former expresses inefficiency in a ratio form, while the latter expresses it in a difference form.

With this relationship, the economic interpretation of Leleu's model also applies to the extended BCC model. The major difference between these two models is that the extended BCC model expresses inefficiency in a ratio form, with one as the lower bound, and whose inverse is conveniently converted to an efficiency measure, while Leleu's model expresses inefficiency in a difference form, with a lower bound of zero and an unknown upper bound, which makes a transformation into an efficiency measure difficult. One situation common to these two models is that they usually have multiple solutions, and it is difficult to identify which ones in this case are the real values perceived by the decision maker or the shadow prices of the factors. 


\section{Envelopment model}

The extended BCC model (3) is slightly simpler than Leleu's model (4). Its dual, which is usually called the envelopment form, is also simpler than that of Leleu's model. The envelopment form of Leleu's model is:

$\max . \delta$

$$
\begin{array}{llr}
\text { s.t. } & \sum_{j=1}^{n} \lambda_{j}\left(Y_{r j}-Y_{r 0}\right) \geq \sigma Y_{r 0}+\delta g_{r}^{Y}, r=1, \ldots, s \\
& \sum_{j=1}^{n} \lambda_{j}\left(B_{k j}-B_{k 0}\right) \leq \sigma B_{k 0}+\delta g_{k}^{B}, k=1, \ldots, t \\
& \sum_{j=1}^{n} \lambda_{j}\left(X_{i j}-X_{i 0}\right) \leq 0, & \\
& \sum_{j=1}^{n} \lambda_{j}+\sigma=1 & \\
& \sigma, \lambda_{j} \geq 0, & j=1, \ldots, m
\end{array}
$$

which is difficult to provide geometric interpretations of, and the technology of variable returns to scale is also difficult to recognize. In contrast, the envelopment form of the extended BCC model, formulated as follows, is better in this aspect:

$$
\begin{array}{lll}
1 / E_{0}=\max . & \eta \\
\text { s.t. } & \sum_{j=1}^{n} \lambda_{j} Y_{r j} \geq \eta Y_{r 0}, & \\
& \sum_{j=1}^{n} \lambda_{j} B_{k j} \leq \eta B_{k 0}, & k=1, \ldots, s \\
& \sum_{j=1}^{n} \lambda_{j} X_{i j} \leq X_{i 0}, \quad & i=1, \ldots, m \\
& \sum_{j=1}^{n} \lambda_{j}=1 & \\
& \lambda_{j} \geq 0, & j=1, \ldots, n
\end{array}
$$

The constraint $\sum_{j=1}^{n} \lambda_{j}=1$ clearly indicates the technology of variable returns to scale.

For geometric interpretations, this model is similar to the conventional $\mathrm{BCC}$ model that treats the undesirable outputs as inputs, except that the undesirable outputs of the unit being evaluated, $B_{k 0}$, are also attached with the distance parameter $\eta$. This is the key feature of this model. Without attaching $\eta$ to $B_{k 0}$, the model implies that $B_{k}$ is strongly disposable, and that it can be disposed of freely, which is unreasonable for an undesirable output. In contrast, there are several merits when $\eta$ is attached to both the desirable and undesirable outputs. First, the model satisfies the condition of weak disposability for outputs. Second, the null-jointness condition that "if $B_{k}=0$ then $Y_{r}=0$ " is satisfied. Third, the model becomes a directional distance one by expressing the right-hand sides of the output constraints, $\eta Y_{r 0}$ and $\eta B_{k 0}, \quad$ as $\quad Y_{r 0}+\quad(\eta-1) Y_{r 0}$ and $B_{k 0}-(\eta-1)\left(-B_{k 0}\right)$, respectively, where the direction is $\left(\boldsymbol{g}^{Y}, \boldsymbol{g}^{B}\right)=\left(\boldsymbol{Y}_{0},-\boldsymbol{B}_{0}\right)$. Therefore, once the desirable outputs are increased, the undesirable outputs must also be increased, and at the same rate.
To illustrate different ideas of efficiency measurement with undesirable outputs, consider five production units, $A, B, C, D$, and $E$, depicted in Figure 1, using one unit of input $X$ to produce different combinations of the desirable output $Y$ and undesirable output $B$. The production frontier constructed under the assumption of strong disposability of outputs is TET', that under weak disposability is OCET, and that by treating the undesirable output as an input is SACES'. The frontier constructed from the extended BCC model is $O C E S$, which is similar to that of weak disposability, except that it extends eastward horizontally beyond Unit $E$, instead of downward vertically. There are three inefficient units, $A, B$, and $D$, under this technology. In measuring the efficiency, for example of Unit $B$, the undesirable output-desirable input exchange approach uses Unit $C$ as the target to result in the efficiency of $Y_{B} / Y_{C}$. However, since the undesirable output is produced with the desirable output, as the desirable output of Unit $B$ increases from the level of $Y_{B}$ to $Y_{C}$, the undesirable output will increase at the same rate to reach point $\hat{B}$, where $Y_{\hat{B}}=Y_{C}$. One cannot dispose of the undesirable output of $\hat{B}$ to that of Unit $C$, and cannot use Unit $C$ as the target to measure efficiency. Unit $B$ in this case must increase both the desirable and undesirable outputs further to reach point $B^{*}$ on the frontier, and use it as the target to obtain the inefficiency of $\eta=O B^{*} / O B$. The idea of Leleu is to use a direction $g$ to reach a point $B^{\circ}$ on the frontier from $B$, and use the absolute difference between $B$ and $B^{\circ}$ as the inefficiency measure $\varphi$.

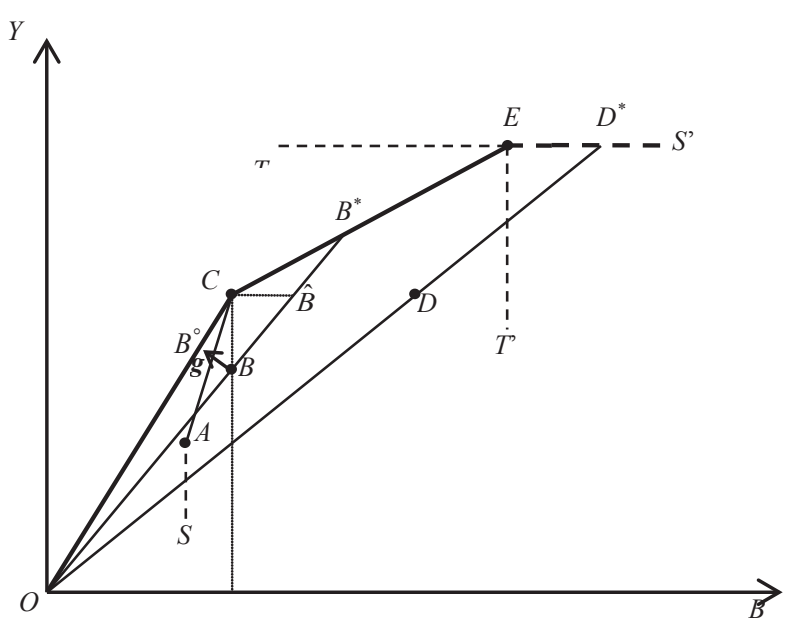

Fig. 1. Frontiers and efficiency measures under different assumptions.

Similar to other BCC-type models, the extended BCC model also faces difficulties in determining appropriate efficiency measures for weakly efficient units, for example $D^{*}$, lying on the horizontal frontier line segment $E S^{\prime}$. A chain effect of this situation is the inappropriate efficiency measure of those units calculated by using the weakly efficient units as the target, for example Unit $D$. The amendment for the BCC-type models is to impose a small non-Archimedean number $\varepsilon$ as the lower bound for the multipliers [4]. When $u_{r}, v_{i}, w_{k} \geq 0$ in constraint set (3.3) of the 
multiplier form of the extended BCC model are replaced with $u_{r}, v_{i}, w_{k} \geq \varepsilon$, the corresponding envelopment model then becomes:

$$
\begin{aligned}
1 / E_{0}=\max . & \eta+\varepsilon\left(\sum_{r=1}^{s} s_{r}^{+}+\sum_{k=1}^{t} t_{k}^{-}+\sum_{i=1}^{m} s_{i}^{-}\right) \\
\text {s.t. } & \sum_{j=1}^{n} \lambda_{j} Y_{r j}-s_{r}^{+}=\eta Y_{r 0}, \quad r=1, \ldots, s \\
& \sum_{j=1}^{n} \lambda_{j} B_{k j}+t_{k}^{-}=\eta B_{k 0}, \quad k=1, \ldots, t \\
& \sum_{j=1}^{n} \lambda_{j} X_{i j}+s_{i}^{-}=X_{i 0}, \quad i=1, \ldots, m \\
& \sum_{j=1}^{n} \lambda_{j}=1 \\
& \lambda_{j} \geq 0, \\
&
\end{aligned}
$$

The value for the lower bound $\varepsilon$ must be appropriately determined to be able to produce proper efficiency measures for those units on the frontier segment ES', and those using them as the target to measure efficiency.

\section{Conclusion}

This paper proposes an extended BCC model to measure the efficiency of production units with undesirable outputs by requiring the associated multipliers to be negative. It is shown that this model is equivalent to the one proposed by Leleu [1], except the inefficiency is expressed in a ratio form, rather than a difference form. Moreover, this model does not have the weaknesses noted by Leleu with regard to modeling undesirable outputs. Specifically, the model has a correct form of variable returns to scale technology, it satisfies the condition of null-jointness, the undesirable outputs are weakly disposable, and the shadow prices of the undesirable outputs are positive. Due to its relationship with Leleu's model, the extended BCC model also shares the economic interpretation of Leleu's model. Its simpler form also makes the geometric interpretation clearer.

The extended BCC model has an advantage that is not possessed by Leleu's model. That is, it produces a relative measure of how far a unit is from the target in the range of $(0,1)$, while Leleu's model gives an absolute distance measure, which cannot be converted to a scale of $(0,1)$ to show a unit's relative performance as compared to other units.

Finally, the idea proposed in Leleu [1] of requiring all units to have the same shadow price for the undesirable output, which is similar to the idea of a common-weight in Kao and Hung [5], can also be applied to the extended BCC model by minimizing the total inefficiency ratios of all units. However, the objective function in this case is nonlinear, and the solution process will be made easier if it can be transformed into a linear one.

\section{References}

1. H. Leleu, Shadow pricing of undesirable outputs in nonparametric analysis. European J. Operational Research 231, 474-480 (2013)

2. R.D. Banker, A. Charnes, W.W. Cooper, Some models for estimating technical and scale efficiencies in data envelopment analysis, Management Science 30, 1078-1092 (1984)

3. A. Charnes, W.W. Cooper, Programming with linear fractional, Naval Research Logistics Quarterly 9, 181-186 (1962)

4. A. Charnes, W.W. Cooper, The non-Archimedean $C C R$ ratio for efficiency analysis: $A$ rejoinder to Boyd and Färe, European J. Operational Research 15, 333-334 (1984)

5. C. Kao, H.T. Hung, Data envelopment analysis with common weights: The compromise solution approach, J. Operational Research Society 56, 1196-1203 (2005) 